Pacific Journal of Mathematics

STRONGLY REGULAR GRAPHS, PARTIAL GEOMETRIES AND 


\title{
STRONGLY REGULAR GRAPHS, PARTIAL GEOMETRIES AND PARTIALLY BALANCED DESIGNS
}

\author{
R. C. Bose
}

0. Summary. This paper introduces the concept of a partial geometry, which serves to unify and generalize certain theorems on embedding of nets, and uniqueness of association schemes of partially balanced designs, by Bruck, Connor, Shrikhande and others. Certain lemmas and theorems are direct generalizations of those proved by Bruck [5], for the case of nets, which are a special class of partial geometries.

1. Introduction. We use graph theoretic methods for the study of association schemes of partially balanced incomplete block (PBIB) designs. For this purpose it is convenient to switch from graph theoretic language to the language of designs and vice versa as necessary.

As we shall be concerned with finite graphs only, we shall use the word graph in the sense of finite graphs.

A graph $G$ with $v$ vertices is said to be regular if each vertex is joined to $n_{1}$ other vertices, and unjoined to $n_{2}$ other vertices. Clearly

$$
v-1=n_{1}+n_{2} \text {. }
$$

If further any two joined vertices of $G$, are both joined to exactly $p_{11}^{1}$ other vertices, and any two unjoined vertices are both joined to exactly $p_{11}^{2}$ other vertices, then the graph $G$ is defined to be strongly regular, with parameters

$$
n_{1}, n_{2}, p_{11}^{1}, p_{11}^{2} \text {. }
$$

The concept of strongly regular graphs is isomorphic with the concept of association schemes of PBIB designs (with two associate classes), which was first introduced by Bose and Shimamoto [4]. Such a scheme they defined as a scheme of relations between $v$ treatements such that

(i) any two objects are either first associates or second associates

Recived August 20,1962. This research was supported in part by the United States Air Force under grant no. AF AFOSR-60-21, monitered by the Office of Scientific Research. The author also wishes to acknowledge, that some of the ideas on which the present paper is based originated during discussions at the Symposium on Combinatorial Mathematics held at the Rand Corporation, Santa Monica, California, in the summer of 1961, in which the author participated. Now a visiting professor to the University of Geneva, on leave from the University of North Carolina. 
(ii) each treatement has $n_{i} i$ th associates $(i=1,2)$

(iii) If two treatments are $i$ th associates, then the number of treatments common to the $j$ th associates of the first and $k$ th associates of the second is $p_{j k}^{i}$ and is independent of the pair of treatments with which we start. Also $p_{j k}^{i}=p_{k j}^{i}$.

Bose and Clatworthy [1] showed that it is unnecessary to assume the constancy of all the $p_{j k}^{i}$ 's. If we assume that $n_{1}, n_{2}, p_{11}^{1}$ and $p_{11}^{2}$ are constant, then the constancy of the $p_{21}^{1}, p_{22}^{1}, p_{12}^{2}, p_{21}^{2}, p_{22}^{2}$ follows and $p_{12}^{1}=p_{21}^{1}, p_{12}^{2}=p_{21}^{2}$.

If we now identify the $v$ treatments of the association scheme with the $v$ vertices of a graph $G$, and consider two vertices as joined or unjoined according as the corresponding treatments are first or second associates, it is clear that a strongly regular graph $G$ with the parameters (1.2) is isomorphic with an association scheme with the same parameters.

We have introduced for the first time in this paper the concept of a partial geometry.

A partial geometry $(r, k, t)$ is a system of undefined points and lines, and an undefined relation incidence satisfying the axioms A1-A4. To avoid cumbersome expression we may use standard geometric language. Thus a point incident with a line may be said to lie on it and the line may be said to pass through the point. If two lines are incident with the same point, we say that they intersect.

A1. Any two points are incident with not more than one line.

A2. Each point is incident with $r$ lines.

A3. Each line is incident with $k$ points.

A4. If the point $P$ is not incident with the line $l$, there pass through $P$ exactly $t$ lines $(t \geqq 1)$ intersecting $l$.

We show that the number of points $v$ and the number of lines $b$ in the partial geometry $(r, k, t)$ are given by

$$
\begin{aligned}
& v=k[(r-1)(k-1)+t] / t, \\
& b=r[(r-1)(k-1)+t] / t .
\end{aligned}
$$

The graph $G$ of a partial geometry is defined as a graph whose vertices correspond to the points of the geometry, and in which two vertices are joined or unjoined according as the corresponding points are incident or non-incident with a common line. We then prove:

THEOREM. The graph $G$ of a partial geometry $(r, k, t)$ is strongly regular with parameters.

$$
\begin{aligned}
& n_{1}=r(k-1), \quad n_{2}=(r-1)(k-1)(k-t) / t, \\
& p_{11}^{1}=(t-1)(r-1)+k-2, \quad p_{11}^{2}=r t .
\end{aligned}
$$


where

$$
1 \leqq t \leqq r, \quad 1 \leqq t \leqq k
$$

Using certain theorems of Bose and Mesner [3] relating to association schemes, we derive:

THEOREM. A necessary condition for the existence of a partial geometry $(r, k, t)$ is that the number

$$
\alpha=\frac{r k(r-1)(k-1)}{t(k+r-t-1)},
$$

is integral.

A strongly regular graph with parameters (1.5), (1.6) and for which (1.7) is satisfied will be defined to be a pseudo-geometric graph with characteristics $(r, k, t)$. Such a graph may or may not be the graph of a partial geometry $(r, k, t)$. It is therefore of interest to study the conditions under which a strongly regular graph, and in particular a pseudo-geometric graph with characteristic $(r, k, t)$ is the graph of a partial geometry $(r, k, t)$.

A subset of vertices of a graph $G$, any two of which are joined is called a clique of $G$. When $G$ is the graph of a partial geometry $(r, k, t)$ there will exist in $G$ a set $\Sigma$ of distinct cliques, $K_{1}, K_{2}, \cdots$, $K_{b}$ corresponding to the lines of the geometry satisfying the following axioms

$A^{*} 1$. Any two joined vertices of $G$ are contained in one and only one clique of $\Sigma$.

A*2. Each vertex of $G$ is contained in $r$ cliques of $\Sigma$.

$\mathrm{A}^{*} 3$. Each clique of $\Sigma$ contains $k$ vertices of $G$.

A*4. If $P$ is a vertex of $G$ not contained in a clique $K_{i}$ of $\Sigma$ there are exactly $t$ vertices in $K_{i}$, which are joined to $P(i=1$, $2, \cdots, l)$.

Hence any graph $G$ in which there exists a set $\Sigma$ of cliques $K_{1}$, $K_{2}, \cdots, K_{b}$, satisfying axioms $\mathrm{A}^{*} 1$ to $\mathrm{A}^{*} 4$, is the graph of a partial geometry $(r, k, t)$. In fact $G$ together with the cliques of $\Sigma$ is isomorphic to a partial geometry $(r, k, t)$ the vertices of $G$ corresponding to the points and the cliques of $\Sigma$ corresponding to the lines of the geometry. Such a graph will be called geometrisable $(r, k, t)$.

One may consider graphs in which there exists a set of cliques $K_{1}, K_{2}, \cdots, K_{b}$ satisfying one or more but not all of the axioms $\mathrm{A}^{*} 1$, $\mathrm{A}^{*} 2, \mathrm{~A}^{*} 3, \mathrm{~A}^{*} 4$. Thus a previous result due to Bose and Clatworthy [1], is equivalent to the following:

THEOREM. If in a strongly regular graph $G$, there exists a set $\Sigma$ of cliques $K_{1}, K_{2}, \cdots, K_{b}$, satisfying the axioms $\mathrm{A}^{*} 1, \mathrm{~A}^{*} 2, \mathrm{~A}^{*} 3$, and 
if $k>r$, then the graph is geometrisable $(r, k, t)$, the vertices of $G$, and the cliques of $\Sigma$ being the points and lines of the corresponding geometry.

We further prove

THEOREM. If in a pseudo-geometric graph with characteristics $(r, k, t)$, there exists a set $\Sigma$ of cliques $K_{1}, K_{2}, \cdots, K_{b}$, satisfying axioms $\mathrm{A}^{*} 1$ and $\mathrm{A}^{*} 2$, and if $k>r$, then $G$ is geometrisable $(r, k, t)$, the vertices of $G$, and the cliques of $\Sigma$ being the points and lines of the corresponding geometry.

There are many interesting examples of partial geometries some of which are given in $\S 7$. In particular the partial geometry $(r, k, t)$ becomes a net of degree $r$ and order $k$ when $t=r-1$. A pseudogeometric graph with characteristics $(r, k, r-1)$ may be defined to be a pseudo-net graph, of degree $r$ and order $k$. Bruck [5] has proved a series of lemmas for pseudo-net graphs and in particular has shown that a pseudo-net graph of degree $r$ and order $k$ is geometrisable $(r, k, r-1)$ if

$$
k>\frac{1}{2}(r-1)\left(r^{3}-r^{2}+r+2\right) .
$$

The special case $r=2$, was proved by Shrikhande [19]. In this paper we give the following generalization of Bruck's result.

THEOREM. A pseudo-geometric graph with characteristics $(r, k$, $t)$ is geometrisable $(r, k, t)$ if

$$
k>\frac{1}{2}\left[r(r-1)+t(r+1)\left(r^{2}-2 r+2\right)\right] \text {. }
$$

We have proved a series of Lemmas which are direct generalizations of the lemmas used by Bruck for his proof. In fact it is surprising how smoothly the technique devised by Bruck for the special case of nets, works in the general case.

In particular the concept of grand cliques introduced by Bruck for the case of nets is capable of easy generalization. If $G$ is a pseudo-geometric graph with characteristics $(r, k, t)$, then a major clique of $G$ is defined as a clique which contains at least $k-(r-1)^{2}$ $(t-1)$ points. A major clique which is also maximal is defined as a grand clique.

Given a pseudo-geometric graph $G$ with characteristics $(r, k, t)$ the set of grand cliques is unambiguously defined. We may take this set to be the set $\Sigma$ and enquire under what circumstances, the axioms $A^{*} 1-A^{*} 4$ will be satisfied. We then show that (1.10) is a sufficient condition for this. 
A pseudo-geometric graph with characteristics $(r, k, t)$ has the same parmeters as the triangular association scheme if we take $r=$ $t=2, k=n-1$. Substituting these values in (1.10) we get $n>8$. Thus for these special values of $r, k, t$ our result is equivalent to the uniqueness of the triangular scheme for $n>8$, a result first proved by Connor [9]. In fact our result may be interpreted as a generalized uniqueness theorem as explained in $\S 12$.

A net of degree $r$ and order $k$ is defined to have deficiency $d=$ $r+1-k$. Bruck [5] showed that a net of order $k$ and deficiency $d$ can be completed to an affine plane by the addition of new lines, if

$$
k>\frac{1}{2}(d-1)\left(d^{3}-d^{2}+d+2\right) .
$$

We generalize Bruck's result to the following:

THEOREM. Given a partially balanced incomplete block (PBIB) design $\left(r, k, \lambda_{1}, \lambda_{2}\right), \lambda_{1}>\lambda_{2}$, based on an association scheme with parameters

$$
\begin{aligned}
& n_{1}=(d-1)(k-1)(k-t) / t, \quad n_{2}=d(k-1), \\
& p_{11}^{1}=[(d-1)(k-1)(k-t)-d(k-t-1)-t] / t, \\
& p_{11}^{2}=(d-1)(k-t)(k-t-1) / t,
\end{aligned}
$$

we can extend the design by adding new blocks, containing the same treatments, in such a way that the extended design is a balanced incomplete block (BIB) design, with $r_{0}=r+d\left(\lambda_{1}-\lambda_{2}\right)$ replications, block size $k$ and in which every pair of treatments occur together in $\lambda_{1}$ blocks.

2. Strongly regular graphs. A finite graph $G$ consists of a finite set of $v$ vertices, and a relation adjacency such that any two distinct vertices of $G$ may be either adjacent or non-adjacent. Adjacent vertices may be said to be joined and non-adjacent vertices to be unjoined. We shall be concerned with finite graphs only, and use the word graph in the sense of finite graphs.

The graph $G$ is said to be regular (of degree $n_{1}$ ) if each vertex of $G$ is joined to exactly $n_{1}$ other vertices. In this case each vertex will be unjoined to exactly $n_{2}$ other vertices, where

$$
n_{1}+n_{2}=v-1 \text {. }
$$

A regular graph $G$ will be said to be strongly regular if (i) any two vertices which are joined in $G$, are both simultaneously joined to exactly $p_{11}^{1}$ other vertices (ii) any two pairs of vertices which are unjoined in $G$, are both simultaneously joined to exactly $p_{11}^{2}$ vertices.

A strongly regular graph $G$ thus depends on four parameters 
$n_{1}, n_{2}, p_{11}^{1}$ and $p_{11}^{2}$, the number of vertices being given by (2.1).

Let two vertices of a strongly regular graph $G$ be called first associates if they are joined, and second associates if they are unjoined. If the vertices $\theta$ and $\phi$ of $G$ are $i$ th associates, we shall denote by $p_{j k}^{i}(\theta, \phi)$ the number of vertices which are $j$ th associates of $\theta$ and $k$ th associates of $\phi$. From definition the number $p_{11}^{i}(\theta, \phi)$ is independent of the pair $\theta, \phi$ so long as they are $i$ th associates. Thus

$$
p_{11}^{i}(\theta, \phi)=p_{11}^{i} \quad i=1,2 .
$$

We shall show that a similar situation prevails with respect to all the numbers $p_{j k}^{i}(\theta, \phi)$, so that we can write

$$
p_{j k}^{i}(\theta, \phi)=p_{j k}^{i} \quad i, j, k=1,2
$$

and that

$$
p_{j k}^{i}=p_{k j}^{i} \text {. }
$$

This follows from the following theorems proved by Bose and Clatworthy [1], in connection with partially balanced incomplete block (PBIB) designs (if we identify treatments with vertices).

THEOREM 2.1. Let there exist a relationship of association between every pair among $v$ treatments satisfying the conditions:

(a) Any two treatments are either first associates or second associates (b) Each treatment has $n_{1}$ first and $n_{2}$ second associates (c) For any pair of treatments which are first associates the number $p_{11}^{1}$ of treatments common to the first associates of the first and the first associates of the second is independent of the pair of treatments with which we start.

Then, for every pair of first associates among the $v$ treatments the number $p_{12}^{1}, p_{21}^{1}$ and $p_{22}^{1}$ are constants, and $p_{12}^{1}=p_{21}^{1}$.

THEOREM 2.2. Let there exist a relationship of association between every pair among $v$ treatments satisfying the conditions:

(a) Any two treatments are either first associates (b) Each treatment has $n_{1}$ first associates and $n_{2}$ second associates (c) For any pair of treatments which are second associates, the number $p_{11}^{2}$ of treatments common to the first associates of the first and the first associates of the second is independent of the pair of treatments with which we start.

Then, for every pair of second associates among the $v$ treatments the numbers $p_{12}^{2}, p_{21}^{2}$ and $p_{22}^{2}$ are constants, and $p_{12}^{2}=p_{21}^{2}$. 
It appears from the proof of Bose and Clatworthy that

$$
\begin{array}{ll}
p_{12}^{1}=n_{1}-p_{11}^{1}-1=p_{21}^{1}, & p_{22}^{1}=n_{2}-n_{1}+p_{11}^{1}+1, \\
p_{12}^{2}=n_{1}-p_{11}^{2}=p_{21}^{2}, & p_{22}^{2}=n_{2}-n_{1}+p_{11}^{2}-1,
\end{array}
$$

Actually the relations (2.3), (2.4) were obtained much earlier by Bose and Nair [3], but in their formulation they started with the constancy of all the numbers $p_{j k}^{i}(i, j, k=1,2)$. It is also easy to prove as shown by Bose and Nair [3], that

$$
n_{1} p_{12}^{1}=n_{2} p_{11}^{2}, \quad n_{1} p_{22}^{1}=n_{2} p_{12}^{2} .
$$

The concept of a strongly regular graph is isomorphic with the concept of an association scheme with two associate classes, as introduced by Bose and Shimamoto [4], in connection with the theory of partially balanced designs, if treatments are identified with vertices, a pair of first associates with a pair of joined vertices, and a pair of second associates with a pair of unjoined vertices. Thus strongly regular graphs first arose in connection with the theory of partially balanced designs.

The numbers $v, n_{1}, n_{2} p_{j k}^{i}$ are called the parameters of the regular graph $G$. They are connected by the relations (2.1)-(2.5), and only four are linearly independent. These may be conveniently chosen as $n_{1}, n_{2}, p_{11}^{1}$ and $p_{11}^{2}$.

3. Partial geometries. Consider two undefined classes of objects called points and lines, together with a relation incidence, such that a point and a line, may or may not be incident. Then the points and lines are said to form a partial geometry $(r, k, t)$ provided that the following axioms are satisfied:

A1. A pair of distinct points is not incident with more than one line.

A2. Each point is incident with exactly $r$ lines.

A3. Each line is incident with exactly $k$ points.

A4. Given a point $P$ not incident with a line $l$, there are exactly $t$ lines $(t \geqq 1)$ which are incident with $P$, and also incident with some point incident with $l$.

If there were two distinct lines $l$ and $m$ each incident with two distinct points $P_{1}$ and $P_{2}$, then A1 would be contradicted. Hence we have,

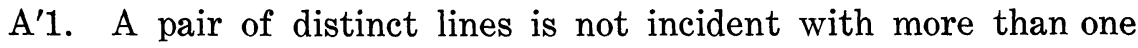
point.

For convenience we will use the ordinary geometric language. Thus if a point is incident with a line, we shall say that the point lies on the line or is contained in the line, and that the line passes 
through the point. If two points are incident with the line, then we speak of the line as joining the two points. By A1 there cannot be more than one line joining two points. Thus either two points are unjoined or joined by a unique line. If two lines are incident with a common point, they are said to intersect, and the common point is said to be their point of intersection. By $A^{\prime} 1$ two lines cannot intersect in more than one point. Hence two lines are either non-intersecting or intersect in a unique point.

Theorem 3.1. Given a partial geometry $(r, k, t)$ there exists a dual partial geometry $(k, r, t)$ obtained by calling points of the first, the lines of the second; and the lines of the first the points of the second.

This follows by noting the duality $\mathrm{A} 1$ and $\mathrm{A}^{\prime} 1$, the duality $\mathrm{A} 2$ and $\mathrm{A} 3$ and the self dual nature of $\mathrm{A} 4$. In fact $\mathrm{A} 4$ can be rephrased as

$\mathrm{A}^{\prime} 4$. Given a line $l$ not incident with a point $P$ there are exactly $t$ points which are incident with $l$ and also incident with some line incident with $P$.

In terms of the alternative geometric language introduced we may write $\mathrm{A} 4$ and $\mathrm{A}^{\prime} 4$ as

A4. Through any point $P$ not lying on a line $l$ there pass exactly $t$ lines intersecting $l$.

$\mathrm{A}^{\prime} 4$. On any line $l$ not passing through a point $P$, there lie exactly $t$ points, joined to $P$.

The equivalence of $\mathrm{A} 4$ and $\mathrm{A}^{\prime} 4$ is now obvious.

4. Graph of a partial geometry. The graph $G$ of a partial geometry $(r, k, t)$ is defined as follows: The vertices of $G$ are the points of the partial geometry. Two vertices of $G$ are joined (adjacent) if the corresponding points of the geometry are joined (incident with the same line). Two vertices of $G$ are unjoined (nonadjacent) if the corresponding points of the partial geometry are unjoined (i.e. there exists no line incident with both the points).

Theorem 4.1. The graph $G$ of partial geometry $(r, k, t)$ is strongly regular with parameters

$$
\begin{gathered}
n_{1}=r(k-1), \quad n_{2}=(r-1)(k-1)(k-t) / t, \\
p_{11}^{1}=(t-1)(r-1)+k-2, \quad p_{11}^{2}=r t \\
1 \leqq t \leqq r, \quad 1 \leqq t \leqq k
\end{gathered}
$$

Let there be $v$ points and $b$ lines in the partial geometry. Since 
the points of the geometry have been identified with the vertices of the graph $G$, we can call two points of the geometry first associates if they are joined by a line, and second associates if they are not joined by a line. Now through any point $P$ of the geometry there pass $r$ lines, each of which contains $k-1$ other points besides $P$. Hence $P$ has exactly $r(k-1)$ first associates. Hence

$$
n_{1}=r(k-1) \text {. }
$$

This shows that $G$ is a regular graph. Consider the $b-r$ lines not passing through $P$. From A4 each of these lines contains exactly $t$ first associates of $P$. Any particular first associate $Q$ of $P$, lies on $r-1$ such lines, since one of the $r$ lines passing through $Q$ joins it to $P$. Hence the number of first associates is

$$
n_{1}=t(b-r) /(r-1) \text {. }
$$

Comparing (4.4) and (4.5) we have

$$
b=r[(r-1)(k-1)+t] / t .
$$

Again each of the $b-r$ lines not passing through $P$ contains exactly $k-t$ second associates of $P$. Any particular second associate $R$ of $P$ lies on $r$ such lines. Hence the number of second associates of $P$ is

$$
n_{2}=(k-t)(b-r) / r .
$$

Substituting for $b$ from (4.6) we have

$$
n_{2}=(r-1)(k-1)(k-t) / t .
$$

Consider any two points $P$ and $Q$ which are first associates. They are joined by a line $l$. We shall count the number of points which are first associates to each of $P$ and $Q$. The $k-2$ points on $l$ other than $P$ and $Q$ are first associates of both $P$ and $Q$. Now there pass $(r-1)$ lines through $P$, other than $l$. By A4 each of these contains $t-1$ first associates of $Q$ other than $P$. Thus these $(t-1)(r-1)$ points are first associates of both $P$ and $Q$. It is easy to see that there are no other first associates of both $P$ and $Q$. Hence

$$
p_{11}^{1}=(t-1)(r-1)+k-2 .
$$

Consider two points $P$ and $R$ which are second associates. There is no line joining them. Each of the $r$ lines passing through $P$ contains $t$ first associates of $R$. Hence

$$
p_{11}^{2}=r t \text {. }
$$


We have now verified the formulae (4.1) and (4.2), showing that $G$ is a strongly regular graph. The values of the other parameters $p_{j k}^{i}$ follow from the identities (2.3) and (2.4). The inequality (4.3) clearly follows from axiom A4.

COROLlaRY. The number of points $v$ and the number of lines: $b$ in a partial geometry $(r, k, t)$ is given by

$$
\begin{aligned}
& v=k[(r-1)(k-1)+t] / t, \\
& b=r[(r-1)(k-1)+t] / t .
\end{aligned}
$$

In view of the isomorphism of association schemes with two associate classes, and strongly regular graphs, the definition of partially balanced incomplete block (PBIB) designs given by Bose and Shimamoto [4], may now be rephrased as follows:

Given a strongly regular graph $G$ with parameters $n_{1}, n_{2}, p_{11}^{1}, p_{11}^{2}$, we may identify its $v$ vertices with $v$ treatments. Then a PBIB design is an arrangement of the $v$ treatments into $b$ sets (called blocks) such that.

$(\alpha)$ Each treatment is contained in exactly $r$ blocks

$(\beta)$ Each block contains $k$ distinct treatments

( $\gamma$ ) Any two treatments which are first associates (joined in $G$ ). occur together in exactly $\lambda_{1}$ blocks. Any two treatments which are second associates (unjoined in $G$ ) occur together in $\lambda_{2}$ blooks

The design may be called a PBIB design $\left(r, k, \lambda_{1}, \lambda_{2}\right)$ based on the strongly regular graph $G$.

Given a partial geometry $(r, k, t)$, with graph $G$, it is clearly a. PBIB design $(r . k, 1,0)$ based on $G$, and this PBIB design is a connected design. This follows because two first associates always occur together in a block, and if two treatments $\theta_{0}$ and $\theta_{2}$ are second associates, we can find a treament $\theta_{1}$ in $p_{11}^{2}=r t>0$ ways, such that $\theta_{0}$ and $\theta_{1}$ are first associates, and $\theta_{1}$ and $\theta_{2}$ are first associates. The incidence matrix of a partial geometry may be defined as the matrix $N=\left(n_{i j}\right)$ where $n_{i j}=1$ if the $i$ th point is incident with the $j$ th line and 0 otherwise. Then $N$ is also the incidence matrix of the corresponding PBIB design. Now Connor and Clatworthy [11] and Bose and Mesner [2], have shown that for a connected PBIB design, with two associate classes, $N N^{\prime}$ has only three distinct characteristic roots, whose multiplicites are $1, \alpha$ and $\beta$ where

$$
\alpha, \beta=\frac{n_{1}+n_{2}}{2} \mp \frac{\left(n_{1}-n_{2}\right)+\gamma\left(n_{1}+n_{2}\right)}{2 \sqrt{\Delta}},
$$

and

$$
\gamma=p_{12}^{2}-p_{12}^{1}, \quad \Delta=\gamma^{2}+2\left(p_{12}^{1}+p_{12}^{2}\right)+1
$$


Now these multiplicities are necessarily integral. Using the formulae (4.1), (4.2), (2.3) and (2.4) we find that

$$
\alpha=\frac{r k(r-1)(k-1)}{t(k+r-t-1)} .
$$

Hence we have the theorem

THEOREM (4.2). A necessary condition for the existence of a partial geometry $(r, k, t)$ is that the number

$$
\alpha=\frac{r k(r-1)(k-1)}{t(k+r-t-1)}
$$

is a positive integer.

5. Partially balanced designs, which are partial geometries. We have already shown that a partial geometry $(r, k, t)$ is isomorphic to a PBIB design $(r, k, 1,0)$ based on the graph (association scheme) of the geometry. However a PBIB design based on a strongly regular graph need not necessarily be a partial geometry. It would therefore be of interest to find sufficient conditions under which a PBIB design $(r, k, 1,0)$ based on a strongly regular graph is isomorphic to a partial geometry.

Now Bose and Clatworthy [1] have shown that if there exists a PBIB design $(r, k, 1,0)$ based on a strongly regular graph $G$ for which $r<k$, then the parameters of $G$ are given by the formulae (4.1), (4.2) i.e. are the same as the parameters of the graph of some partial geometry $(r, k, t)$. We shall show that the design is indeed a partial geometry and thus establish the following theorem:

TheOREM (5.1). If there exists a PBIB design $(r, k, 1,0)$ based on a strongly regular graph $G$, then if $r<k$, the design must be a partial geometry $(r, k, t)$ for some $t \leqq r$. The parameters of $G$ are given by the formulae (4.1), (4.2).

The parameters of $G$ are given by (4.1), (4.2) in view of the result of Bose and Clatworthy already cited. Also the axioms A1, A2, A3 for a partial geometry are implicit in the definition of a PBIB design $(r, k, 1,0)$. It therefore only remains to prove axiom $\mathrm{A} 4$ which amounts to saying that each block of the design contains exactly $t$ treatments which are first associates of a given treatment not contained in the block.

Let $K$ be the set of $k$ treatments contained in a particular block, and let $\bar{K}$ be the set of the remaining $v-k$ treatments. Let $g(x)$ denote the number of treatments in $\bar{K}$ which have exactly $x$ first associates in $K$. Then 


$$
\sum_{x=0}^{k} g(x)=v-k=k(k-1)(r-1) / t .
$$

Let us count the number of pairs $(P, Q)$, where $P$ is a treatment in $K, Q$ is a treatment in $\bar{K}$, and $P$ and $Q$ are first associates. Now each treatment in $K$ has $k-1$ first associates in $K$, and consequently $n_{1}-k+1$ first associates in $\bar{K}$. Hence the required number is $k\left(n_{1}-k+1\right)$. Again there are $g(x)$ treatments in $\bar{K}$, which have exactly $x$ treatments in $K$. These treatments contribute $x g(x)$ to our count. Hence

$$
\sum_{x=0}^{k} x g(x)=k\left(n_{1}-k+1\right)=k(r-1)(k-1) .
$$

Again let us count the number of triplets $\left(P_{1} P_{2}, Q\right)$ where $P_{1} P_{2}$ is an ordered pair of distinct treaments in $K$, and $Q$ is a treatment in $\bar{K}$ which is a first associate of both $P_{1}$ and $P_{2}$. Since $P_{1}$ and $P_{2}$ have $k-2$ common first associates in $K$, they have $p_{11}^{1}-k+2$ common first associates in $\bar{K}$. Hence the required number of triplets like $\left(P_{1} P_{2}, Q\right)$ is $k(k-1)\left(p_{11}^{1}-k+2\right)$. Now each of the $g(x)$ treatments in $\bar{K}$, which have $x$ first asscciates in $K$ contribute $x(x-1) g(x)$ to our count. Hence we have the equation

$$
\begin{aligned}
\sum_{x=0}^{k} x(x-1) g(x) & =k(k-1)\left(p_{11}^{1}-k+2\right) \\
& =k(k-1)(t-1)(r-1) .
\end{aligned}
$$

Using (5.1), (5.2) and (5.3) a simple calculation shows that

$$
\bar{x}=\Sigma x g(x) / \Sigma g(x)=t,
$$

i.e. the average value of $x$ (the number of first associates in $K$ of any treatment of $\bar{K}$ is $t$. Also

$$
\sum_{x=0}^{k} g(x)(x-t)^{2}=0,
$$

which shows that $x$ must always have the value $t$. This proves our theorem.

6. Geometrisable and pseudo-geometric graphs. A strongly regular graph $G$ which has parmeters (4.1) and (4.2) and for which the inequality (4.3) is satisfied, is defined to be a pseudo-geometric graph with characteristics $(r, k, t)$. Thus a pseudo-geometric graph with characteristics $(r, k, t)$ has the same parameters as the graph of a partial geometry $(r, k, t)$. However a graph may be pseudogeometric without being the graph of a partial geometry.

A subset of vertices of a graph $G$, any two of which are joined 
is called a clique of $G$. When $G$ is the graph of a partial geometry there will exist in $G$ a set $\Sigma$ of distinct cliques $K_{1}, K_{2}, \cdots, K_{b}$, corresponding to the lines of the geometry satisfying the following axioms;

A*1. Any two joined vertices of $G$ are contained in one and only one clique of $\Sigma$.

$\mathrm{A}^{*}$. Each vertex of $G$ is contained in $r$ cliques of $\Sigma$.

A*3. Each clique of $\Sigma$ contains $k$ vertices of $G$.

$\mathrm{A}^{*} 4$. If $P$ is a vertex of $G$ not contained in a clique $K_{i}$ of $\Sigma$, there are exactly $t$ vertices in $K_{i}$ which are joined to $P(i=1$, $2, \cdots, b)$.

Hence any graph $G$ in which there exists a set $\Sigma$ of cliques $K_{1}, K_{2}, \cdots, K_{b}$, satisfying axioms $\mathrm{A}^{*} 1-\mathrm{A}^{*} 4$ is the graph of a partial geometry $(r, k, t)$. In fact $G$ together with the cliques of $\Sigma$ is isomorphic to a partial geometry $(r, k, t)$, the vertices of $G$ corresponding to the points, and cliques of $\Sigma$ to the lines of the geometry. Such a graph will be said to be geometrisable $(r, k, t)$.

One may consider graphs in which there exist a set $\Sigma$ of cliques $K_{1}, K_{2}, \cdots, K_{b}$ satisfying one or more but not all of the axioms $\mathrm{A}^{*} 1$, $\mathrm{A}^{*} 2, \mathrm{~A}^{*} 3, \mathrm{~A}^{*} 4$, and investigate under what additional conditions the graph will be geometrisable. Thus theorem (5.1) may be rephrased as

Theorem (6.1). If there esists a set $\Sigma$ of cliques $K_{1}, K_{2}, \cdots, K_{b}$ in a strongly regular graph $G$, satisfying axioms $\mathrm{A}^{*} 1, \mathrm{~A}^{*} 2, \mathrm{~A}^{*} 3$ and if $k>r$, then $G$ is geometrisable $(r, k, t)$.

THeOREM (6.2). Let $G$ be a pseudo-geometric graph with characteristics $(r, k, t)$. If it is possible to find in $G$ a set $\Sigma$ of cliques $K_{1}, K_{2}, \cdots, K_{b}$, satisfying axioms $\mathrm{A}^{*} 1$ and $\mathrm{A}^{*} 2$, and if $k>r$, then $G$ is geometrisable $(r, k, t)$.

We shall prove that each of the cliques $K_{1}, K_{2}, \cdots, K_{b}$ contains exactly $k$ vertices, and that if $Q$ is any vertex not contained in any clique $K_{i}(1 \leqq i \leqq b)$, then there are exactly $t$ vertices in $K_{i}$ which are first associates of (joined to) $Q$. This will show that if the vertices of $G$ are taken as points, and the cliques $K_{1}, K_{2}, \cdots, K_{b}$ as lines, then we have a partial geometry $(r, k, t)$.

Let $P$ be any vertex. Without loss of generality we can take $K_{1}, K_{2}, \cdots, K_{r}$ to contain $P$. Now the sets $K_{1}-P, K_{2}-P, \cdots, K_{r}-$ $P$ are disjoint and must contain between them all the $r(k-1)$ first associates of $P$. From this it follows that the average number of vertices in the $r$ cliques of the set $K_{1}, K_{2}, \cdots, K_{r}$ is $k$. Hence there exists a clique containing at least $k$ vertices. Let $K_{j}$ be such a clique. Let us take a subset $K$ of $K_{j}$, such that $K$ contains ex- 
actly $k$ vertices. Let $\bar{K}$ be the of vertices not contained in $K$. Let $g(x)$ be the number of vertices in $\bar{K}$ which have exactly $x$ first associates in $K$. Then it follows exactly as in the proof of Theorem (5.1) that

$$
\sum_{x=0}^{k} g(x)=k(k-1)(r-1) / t
$$

and

$$
\sum_{x=0}^{k} x g(x)=k(r-1)(k-1) .
$$

Hence the average value of $x$ is $t$. Also as before

$$
\sum_{x=0}^{k}(x-t)^{2} g(x)=0,
$$

which is only possible if $x$ is constant and equal to $t$. Hence every point of $\bar{K}$ has exactly $t$ first associates in $K$.

If $K_{j}$ contains any vertex $Q$ other than those already contained in $K$, then $Q$ belongs to $\bar{K}$, and therefore has, exactly $t$ first associates in $K$. But each point of $K$ is a first associate of $Q$ hence $t=k$, which contradicts $1 \leqq t \leqq r<k$. This shows that none of the cliques $K_{j}$ containing $P$, contains more than $k$ vertices. Thus each contains exactly $k$ vertices. Since each clique contains at least one vertex, each of the clique $K_{1}, K_{2}, \cdots, K_{b}$ contains exactly $k$ vertices. Also if $Q$ is a point not contained in $K_{i}(1 \leqq i \leqq b)$, then there are exactly $t$ vertices in $K_{i}$ which are first associates of $Q_{i}$. This completes the proof of the theorem.

N. B. Compare Theorems (6.1) and (6.2).

7. Examples of partial geometries. (a) A net $(r, k)$ of degree $r$ and order $k$ is a system of undefined points and lines together with an incidence relation subject to the following axioms (i) There is at least one point (ii) The lines of the net can be partitioned into $r$ disjoint, nonempty, "parallel classes" such that each point of the net is incident with exactly one line of each class and given two lines belonging to distinct classes there is exactly one point of the net which is incident with both lines.

For convenience we can use phrases such as "point is on a line" istead of speaking of incidence. Then it can be readily proved (see for example Bruck [5]) that

(1) Each line of the net contains exactly $k$ distinct points where $k \geqq 1$.

(2) Each point of the net lies on exactly $r$ distinct lines where $r \geqq 1$. 
(3) The net has exactly $r k$ distinct lines. These lines fall into $r$ parallel classes of $k$ lines each. Distinct lines of the same parallel class have no common points. Two lines of different classes have one common point.

(4) The net has exactly $k^{2}$ distinct points.

We shall show that $a$ net $(r, k)$ of degree $r$ and order $k$ is a partial geometry $(r, k, r-1)$. The properties (1) and (2) above show that axioms A3 and A2 of a partial geometry hold.

Two lines cannot intersect in more than one point, for they either belong to the same parallel class and have no common point, or different parallel classes in which they have one common point. Form this follows the fact two distinct points cannot be incident with more then one line. Hence axiom A1 for a partial geometry holds.

Again given a point $P$ not incident with the line $l$. There are exactly $r$ lines through $P$, one belonging to each parallel class. One of these is parallel to $l$ (i.e. belongs to the same parallel class as $l$ ), and ones not intersect $l$. The other $r-1$ lines through $P$ each intersects $l$ in one point, these points being all distinct. Hence axiom A4 for a partial geometry holds with $t=r-1$. This completes the proof of our statement.

It follows from Theorem 4.1, that the parameters of the graph $G_{N}$ of a net $(r, k)$ are given by

$$
\begin{aligned}
& n_{1}=r(k-1), \quad n_{2}=(k-1)(k-r+1), \\
& p_{11}^{1}=(r-2)(r-1)+(k-2), \quad p_{11}^{2}=r(r-1) .
\end{aligned}
$$

If a strongly regular graph has parameters (7.1), (7.2) we shall call it a pseudo-net graph with characteristic $(r, k)$. A pseudo-net graph with characteristics $(r, k)$ is pseudo-geometric with characteristics $(r, k, r-1)$.

Bruck [5], defines the deficiency $d$ of a net $(r, k)$ by

$$
d=k-r+1 \text {. }
$$

The interpretation of the deficiency $d$ is that if it were possible to add $d$ more parallel classes, each consisting of $k$ lines, so that the extended net now has $k+1$ classes of parallels, the net would become an affine plane, in which any two points are joined by a unique line.

If we take the $k^{2}$ points of the net as treatments and the rk lines as blocks, we obtain what is known as the lattice design. This is a PBIB design $(r, k, 1,0)$ based on the strongly regular graph $G_{N}$ with parameters given by (7.1), (7.2). Lattice designs were introduced by Yates [24]. The association scheme corresponding to $G_{N}$ is 
the $L_{r}$ scheme defined by Bose and Shimanoto [4].

It is well known that a latice design with $r$ replications and block size $k$ is equivalent to a system of $r-2$ mutually orthogonal Latin squares of order $k$.

If $r-2$ mutually orthogonal Latin square of order $k$ are given, we can superpose them. Then each cell contains $r-2$ symbols belonging in order to the different Latin squares. The $k^{2}$ cells are now identified with $k^{2}$ treatments. Treatments belonging to the same row give one set of $k$ blocks. Treatments bolonging to the same column give another set of $k$ blooks. Treatments (cells) which contain the same symbol of the $i$ th Latin square give a set of blocks for each value of $i(i=1,2, \cdots, r-2)$. We thus get $r$ sets of blocks. The treatments and blocks so obtained constitute a lattice design.

Conversely given a Lattice design with $r$ replications and block size $k$, we can construct a set of $r-2$ mutually orthogonal Latin squares of order $k$.

If $r-2$ mutually orthogonal Latin squares of order $k$ are given, we can superpose them. Then each cell contains $r-2$ symbols belonging in order to the different Latin squares. The $k^{2}$ cells are now identified with $k^{2}$ treatments. Treatments belonging to the same row give one set of $k$ blocks. Treatments belonging to the same column give another set of $k$ blocks. Treatments (cells) which contain the same symbol of the $i$ th Latin square give a set of blocks for each value of $i(i=1,2, \cdots, r-2)$. We thus get $r$ sets of blocks. The treatments and blocks so obtained constitute a lattice design.

Conversely given a Lattice design with $r$ replications and block size $k$, we can construct a set of $r-2$ mutually orthogonal Latin squares of order $k$.

(b) Take an $n \times n$ squares and write down the numbers $1,2, \cdots$, $n(n-1) / 2$ in the cells above the main diagonal. Fill up the cells below the main diagonal symmetrically. The case $n=5$ is exemplified below.

\begin{tabular}{|c|c|c|c|c|}
\hline$*$ & 1 & 2 & 3 & 4 \\
\hline 1 & $*$ & 5 & 6 & 7 \\
\hline 2 & 5 & $*$ & 8 & 9 \\
\hline 3 & 6 & 8 & $*$ & 10 \\
\hline 4 & 7 & 9 & 10 & $*$ \\
\hline
\end{tabular}

Fig. 1

The cells containing the same number are identified with the same point. Thus there are two different cells representing the same 
point, there being $v=n(n-1) / 2$ points all together. Let the $n$ rows constitute lines. Thus there are $n$ lines. It is clear that axioms $\mathrm{A} 1, \mathrm{~A} 2, \mathrm{~A} 3$ of a partial geometry are satisfied with $r=2, k=n-1$. It is easy to see that any two lines intersect in one point. Thus $t=2$, and we have a partial geometry $(2, n-1,2)$.

If two points which lie on a line are called first associates, and two points which do not lie on any line are called second associates, we have the triangular association scheme first defined by Bose and Shimamoto [4], and extensively studied by Connor [9], Shrikhande [21], Hoffman [13, 14] and Chang [6, 7]. The parameters of the association scheme or the corresponding strongly regular graph are

$$
\begin{array}{ll}
n_{1}=2(n-2), & n_{2}=(n-2)(n-3) / 2, \\
p_{11}^{1}=n-2, & p_{11}^{2}=4 .
\end{array}
$$

If a strongly regular graph has the parameters (7.4), (7.5) we shall call it a pseudo-triangular graph with characteristic $n$. A pseudo-triangular graph with characteristic $n$ is pseudo-geometric with characteristics $(2, n-1,2)$.

(c) A balanced incomplete block design BIB is an arrangement of a set of $v_{0}$ objects or treatments in $b_{0}$ sets or blocks, such that (i) each block contains $k_{0}$ distinct treatments (ii) each treatment is contained in $r_{0}$ blocks (iii) each pair of distinct treatments is contained in $\lambda_{0}$ blocks. This design has sometimes been called a $\left(v_{0}, k_{0}\right.$, $\lambda_{0}$ ) configuration. The dual of a design is defined as a new design whose treatments and blocks are in $(1,1)$ correspondence with the blocks and treatments of the original design, and incidence is preserved (where a block and a treatment are incident if the treatment is contained in the block, and non-incident otherwise), Shrikhande [23] has shown that the dual of a BIB design with $\lambda_{0}=1$ is a PBIB design. Now a BIB design with $\lambda_{0}=1$ is clearly a partial geometry $\left(r_{0}, k_{0}, k_{0}\right)$. Hence the dual design is the dual partial geometry $\left(k_{0}, r_{0}, k_{0}\right)$. If we set $k_{0}=r$ and $r_{0}=k$, so as to make $r$ the replication number and $k$ the block size in the dual design, then the dual design is the partial geometry $(r, k, r)$. Any two blocks (lines) of this design intersect in a unique treatment (point). Hence the association scheme of this design has been called the SLB (singly linked block) scheme by Bose and Shimamoto [4]. The parameters of the corresponding strongly regular graph can be written down directly from Theorem (4.1). We have

$$
\begin{aligned}
& n_{1}=r(k-1), \quad n_{2}=(k-r)(r-1)(k-1) / r, \\
& p_{11}^{1}=(r-1)^{2}+k-2, \quad p_{11}^{2}=r^{2} .
\end{aligned}
$$

If a strongly regular graph has the parameters (7.6), (7.7) we 
shall call it a pseudo-SLB graph with characteristics $(r, k)$. A pseudoSLB graph with characteristics $(r, k)$ is a pseudo-geometric graph with characteristics $(r, k, r)$.

(d) To conclude we shall give a rather less obvious example of a partial geometry.

Consider an elliptic non-degenerate quadric $Q_{5}$ in the finite projective space $P G\left(5, p^{n}\right)$. This quadric is ruled by straight lines, called generators, but contains no plane. As shown by Primrose [16] and Ray-Chaudhuri [17], there are $\left(s^{3}+1\right)(s+1)$ points and $\left(s^{3}+1\right)\left(s^{2}+1\right)$ generators in $Q_{5}$, each generator contains $s+1$ points, and through each point there pass $s^{2}+1$ generators, where $s=p^{n}$.

If $P$ is a point on $Q_{5}$ not contained in a generator $l$, then the polar 4 -space of $P$ intersects $l$ in a single point $P^{*}$, and $P P^{*}$ is a generator of $Q_{5}$. It can be readily verified by using theorems proved by Ray-Chaudhuri that $P P^{*}$ is the only generator through $P$, which intersects $l$. This shows that if we consider the points and generators of $Q_{5}$ as points and lines, they constitute a partial geometry $\left(s^{2}+1, s+1,1\right)$. The parameters of the graph of this partial geometry can be easily written down using Theorem (4.1). They are

$$
\begin{array}{ll}
n_{1}=s\left(s^{2}+1\right), & n_{2}=s^{4}+1, \\
p_{11}^{1}=s-1, & p_{11}^{2}=s^{2}+1 .
\end{array}
$$

The partial geometry $\left(s^{2}+1, s+1,1\right)$ is of course a PBIB design. This was obtained by Ray-Chaudhuri [18], the special case $s=2$ was given earlier by Bose and Clatworthy [1]. An interesting point in the present formulation is that to verify that the configuration of points and generators on $Q_{5}$ is a PBIB design we have only to check the constancy of $r, k$ and $t$ instead of the constancy of $r, k, n_{1}, n_{2}$, $p_{11}^{1}, p_{11}^{2}$ as was done by Bose and Clatworthy [1] and by Ray-Chaudhuri [18]. The dual partial geometry $\left(s+1, s^{2}+1,1\right)$ is also of interest.

In the same way one can show that the configuration of points and generators on a non-degenerate quadric $Q_{4}$ in $P G\left(4, p^{n}\right)$ is a partial geometry $(s+1, s+1,1)$ where $s=p^{n}$. The corresponding design was first obtained by Clatworthy [8].

8. Lemmas on claws in pseudo-geometric graphs. We have shown in Theorem (5.1), that if we can base a PBIB design $(r, k, 1,0)$ on a strongly regular graph $G$, then $G$ is pseudo-geometric and the design is a partial geometry. We can ask the converse question: If the graph $G$ is pseudo-geometric $(r, k, t)$ can we base a PBIB design $(r, k, 1,0)$ on it? In graph theoretic language this question may be put as: If the graph $G$ is pseudo-geometric $(r, k, t)$ can we find a set of cliques $K_{1}, K_{2}, \cdots, K_{b}$ satisfying the axioms $\mathrm{A}^{*} 1-\mathrm{A}^{*} 4$ of $\S 6$. 
In the rest of the paper we shall frequently use the following functions:

$$
\begin{aligned}
& \gamma(r, t)=1+(r-1)^{2}(t-1), \\
& q(r, t)=1+(r-1)(2 r-1)(t-1), \\
& \rho(r, t)=r t+(r-1)(t-1)(2 r-1), \\
& p(r, t)=\frac{1}{2}\left[r(r-1)+t(r+1)\left(r^{2}-2 r+2\right)\right] .
\end{aligned}
$$

We note that in view of the inequality $1 \leqq t \leqq r$

$$
p(r, t) \geqq \rho(r, t) \geqq q(r, t) \geqq \gamma(r, t) .
$$

The concept of a claw was suggested by Alan Hoffman in conversation with $R$. H. Bruck and the author. By a claw $[P, S]$ of a pseudo-geometric graph $G$ is meant an ordered pair consisting of a vertex $P$, the vertex of the claw, and a nonempty set $S$ of vertices distinct from $P$ such that every vertex in $S$ is joined to $P$ in $G$ but no two vertices in $S$ are joined in $G$.

The number of elements in a finite set $S$ will be denoted by $|S|$. The order of the claw $[P, S]$ is defined as $s=|S|$.

In Lemmas 8.1-8.3, $G$ denotes a pseudo-geometric graph $(r, k, t)$.

LEMmA 8.1. If $k>\gamma(r, t)=1+(t-1)(r-1)^{2}$ then for any $s$, $1 \leqq s \leqq r$, each vertex $P$ of $G$ is the vertex of a claw $[P, S]$ of order $s$. We can choose $S$ to include any vertex $A$ joined to $P$ in $G$.

Let $P$ be a vertex of $G$. Suppose there exists a claw $[P, S]$ of order $s$. Let $T$ be the set of all vertices other than those belonging to $[P, S]$ and which are joined to $P$ (are first associates of $P$ ). Let $f(x)$ be the number of vertices $Q$ in $T$, such that $Q$ is joined to exactly $x$ vertices in $S(f(x)$ is the number of vertices in $T$ each of which has $f(x)$ first associates in $S$ ). Then we have

$$
\sum_{x=0}^{s} f(x)=n_{1}-s=r(k-1)-s,
$$

since the left hand side of (8.6) counts all first associates of $P$, which are not in $S$. Now let us count pairs $(A, Q)$ where $A$ is in $S$ and $Q$ is in $T$ and is a first associate of both $A$ and $P$. Since $A$ and $P$ have exactly $p_{11}^{1}$ common first associates (none of which can belong to $S$ by the definition of a claw) we have $s p_{11}^{1}$ pairs like $(A, Q)$.

Again there are $f(x)$ vertices in $T$ each of which has exactly $x$ first associates in $S$. They contribute $x f(x)$ to our count, Hence

$$
\sum_{x=0}^{s} x f(x)=s p_{11}^{1}=s\{(t-1)(r-1)+(k-2)\} .
$$


Hence

$$
f(0)-\sum_{x=1}^{s}(x-1) f(x)=(r-s)(k-1)-s(t-1)(r-1) .
$$

Hence if $s<r$ and $k>\gamma(r, t)=1+(r-1)^{2}(t-1)$, then $f(0)$ is positive, i.e. there is at least one vertex $A_{s+1}$ in $T$ which is not joined to $A_{1}, A_{2}, \cdots, A_{s}$. We can therefore add $A_{s+1}$ to $S$ and get a claw of order $s+1$. In this way we can go on extending a claw till we get a claw of any required order not exceeding $r$. We can start this process with any claw $[P, A]$ of order 1 . This proves the lemma.

Lemma (8.2). If $k>\gamma(r, t)=1+(r-1)^{2}(t-1)$, and if $[P, S]$ is a claw of order $r-1$, then there exist at least $k-(r, t)$ distinct vertices $Q$ of $G$ such that $[P, S \cup Q]$ is a claw of order $r$.

If $[P, S]$ is a claw of order $r-1$, then from (8.8)

$$
f(0) \geqq k-\gamma(r, t) .
$$

Hence there exist at least $k-\gamma(r, t)$ vertices $Q$, each of which taken together with $S$ give a claw $\left[P, S^{*}\right]$ of order $r$ where $S^{*}=$ $S \cup Q$.

LEMMA (8.3). If $k>p(r, t)=\frac{1}{2}\left[r(r-1)+t(r+1)\left(r^{2}-2 r+2\right)\right]$ then there exists in $G$ no claw of order $r+1$.

Let $[P, S]$ be a claw of order $s$ in $G$. Let the set $T$ be as in Lemma (8.1). We shall count the number of triplets $\left(A_{1} A_{2}, Q\right)$ where $A_{1}, A_{2}$ is an ordered pair of distinct vertices in $S$, and $Q$ is a vertex in $T$ which is a first associate of both $A_{1}$ and $A_{2}$. Since $A_{1}$ and $A_{2}$ are second associates they have exactly $p_{11}^{2}-1$ common first associates other than $P$. Some of these may not lie in $T$. Hence an upper bound for the required number of triplets is $s(s-1) p_{11}^{2}$. However the $f(x)$ vertices in $T$, each of which has exactly $x$ first associates. in $S$, contribute $x(x-1) f(x)$ to our count. Hence

$$
\sum_{x=0}^{s} x(x-1) f(x) \leqq s(s-1)\left(p_{11}^{2}-1\right)=s(s-1)(r t-1) .
$$

If possible let $s=r+1$. Then adding (8.8) to (8.9) multiplied by a half, and noting that on the left hand side of (8.9) the term $x=0$ contributes nothing we get

$$
\begin{aligned}
f(0) & +\frac{1}{2} \sum_{x=1}^{s}(x-1)(x-2) f(x) \\
& \leqq-k+\frac{1}{2}\left[r(r-1)+t(r+1)\left(r^{2}-2 r+2\right)\right] .
\end{aligned}
$$


Hence if $k>p(r, t)$, there cannot exist a claw of order $r+1$, as the left hand side is essentially positive.

9. Lemmas on cliques in pseudo-geometric graphs. In the Lemmas (9.1) to (9.6), $G$ denotes a pseudo-geometric graph $(r, k, t)$. The definition of a major clique generalizes Bruck's definition, and the concept of a grand clique is taken over from Bruck [5].

A major clique $K$ of $G$ is a clique such that

$$
|K| \geqq k+1-\gamma(r, t)=k-(r-1)^{2}(t-1) .
$$

A grand clique is a major clique which is also a maximal clique.

If $K$ and $L$ are distinct maximal cliques, then $K \cup L$ cannot be a clique. Since $K$ and $L$ are distinct, there must be a vertex $P$ in one of them (say $K$ ), not belonging to the other $(L)$. Now if $K \cup L$ is a clique, then $P$ is joined to every vertex of $L$. Thus $P \cup L$ is a clique which contradicts the fact that $L$ is maximal. Since grand cliques are maximal the union of two grand cliques cannot be a clique.

If we take the set of grand cliques as the set $\Sigma$ of $\S 6$, we may enquire under what conditions the axioms $A * 1, A^{*} 2, A^{*} 3$ and $A^{*} 4$ are satisfied. The lemmas which follow are directed to this purpose.

Lemma (9.1). If $k>(r, t)$ and if $G$ has no claw of order $r+1$, then for every pair of distinct joined vertices $P$ and $Q$ in $G$, there exists at least one major clique containing both $P$ and $Q$.

From Lemma (8.1) we can find a claw $[P, S]$ of order $r$ such that $Q \in S$. Let $A_{1}, A_{2}, \cdots, A_{r-1}$ be other vertices of $S$. Let $\Omega$ be the set of vertices $R$ which when adjoined to $S$, give a claw $P, S^{*}$ of order $r, S^{*}=S \cup R$. From Lemma (8.2), the number of points in $\Omega=|\Omega| \geqq k-\gamma(r, t)$.

The vertices in $\Omega$ are all joined to one another. If any two were not joined they could be added to $A_{1}, A_{2}, \cdots, A_{r-1}$ to give a claw of order $r+1$. Thus $P$ and the vertices in $\Omega$ are all mutually joined. Hence $P \cup \Omega=K$ is a clique of order $\geqq k+1-\gamma(r, t)$, i.e. a major clique.

CoRollary 1. In Lemma (9.1) the hypothesis may be replaced by $k>p(r, t)$.

This follows from Lemma (8.3) by noting that $p(r, t) \geqq \gamma(r, t)$ for $1 \leqq t \leqq r$.

CoRollary 2. When the conditions of Lemma (9.1) or Corollary 1 are satisfied, $P$ and $Q$ are contained in at least one grand clique. 
We can extend the major clique $K$ by adding new vertices till it becomes maximal, and therefore a grand clique.

Lemma (9.2). If $K$ and $L$ are cliques of $G$ and $K \cup L$ is not a clique then $|K \cap L| \leqq r t$.

Since $K \cup L$ is not a clique, there must exist in $K \cup L$ a pair of vertices $P_{1}, P_{2}$ not joined to one another such that $P_{1} \in K, P_{2} \in L$. Any vertex belonging to $|K \cap L|$ must be joined to both $P_{1}$ and $P_{2}$. Hence the number of vertices in $|K \cap L|$ cannot exceed $p_{11}^{2}$. Thus

$$
|K \cap L| \leqq r t \text {. }
$$

Lemma (9.3). If $K$ and $L$ are cliques of $G$ and $K \cap L$ contains at least two vertices $A$ and $B$, then

$$
|K \cup L| \leqq k+(r-1)(t-1) .
$$

Every vertex in $K \cup L$, other than $A$ and $B$, is a first associate of both $A$ and $B$. Hence

$$
|K \cup L| \leqq p_{11}^{1}+2=k+(r-1)(t-1) .
$$

Lemma (9.4). If $K$ and $L$ are cliques of $G$ such that (i) $K \cup L$ is not a clique (ii) $K \cap L$ contains at least two vertices, then

$$
|K|+|L| \leqq k+r t+(r-1)(t-1) .
$$

Since conditions of Lemmas (9.2) and (9.3) are satisfied

$$
\begin{aligned}
|K|+|L|= & |K \cap L|+|K \cup L| \\
& \leqq r t+k+(r-1)(t-1) .
\end{aligned}
$$

LEMMA (9.5). If $k>\rho(r, t)=r t+(r-1)(t-1)(2 r-1)$ and if $G$ has no claw of order $r+1$, then two distinct vertices of $G$ which are joined, are contained in one and only one grand clique.

Suppose there are two distinct grand cliques $K$ and $L$ both containing $P$ and $Q$. Then $K \cup L$ cannot be a clique. Also $K \cap L$ has at least two vertices $P$ and $Q$. Hence from Lemma (9.4),

$$
|K|+|L| \leqq r t+k+(r-1)(t-1) .
$$

Since $K$ and $L$ are grand cliques, they are both major. Hence

$$
\begin{gathered}
2\left[k-(r-1)^{2}(t-1)\right] \leqq|K|+|L| \leqq r t+k+(r-1)(t-1) \\
k \leqq r t+(r-1)(t-1)(2 r-1)=\rho(t),
\end{gathered}
$$


which is a contradiction.

COROLLARY. In Lemma (9.5) the hypothesis may be replaced by $k>p(r, t)$.

LEMmA (9.6) If (i) $k>q(r, t)=1+(r-1)(2 r-1)(t-1)$; (ii) two distinct vertices of $G$ are contained in utmost one grand clique of $G$, (iii) there exists no claw of order $r+1$ in $G$, then each point of $G$ is contained in exactly $r$ grand cliques.

From Lemma (9.1), Corollary 2 any two vertices are contained in at least one grand clique. It follows from assumption (ii), that any two vertices of $G$ are contained in one and only one grand clique. Again from Lemma (8.1), there exists a claw $[P, S]$ of order $r$, where $S=\left\{A_{1}, A_{2}, \cdots, A_{r}\right\} . \quad$ As in Lemma (8.1), let $T$ be the set of first associates of $P$, other than $A_{1}, A_{2}, \cdots, A_{r}$. We define $H_{j}$ as the set consisting of $P, A_{j}$ and all $Q \in T$, such that $Q$ is a first associate of $A_{j}$ but not of $A_{i}$ when $i \neq j$. Let $f(x)$ be as in Lemma (8.1). Now $f(0)=0$, since there are no claws of order $r+1$. Hence from (8.6) and (8.7) we have

$$
\begin{aligned}
& \sum_{x=1}^{r} f(x)=n_{1}-r=r(k-2), \\
& \sum_{x=1}^{r} x f(x)=r p_{11}^{1}=r(k-2)+r(r-1)(t-1), \\
& \sum_{x=2}^{r}(x-1) f(x)=r(r-1)(t-1) .
\end{aligned}
$$

Now

$$
\begin{aligned}
& \sum_{2}^{r} f(x) \leqq \sum_{2}^{r}(x-1) f(x) \leqq(r-1) \sum_{2}^{r} f(x) . \\
& r(t-1) \leqq \sum_{2}^{r} f(x) \leqq r(r-1)(t-1) . \\
& r(t-1) \leqq r(k-2)-f(1) \leqq r(r-1)(t-1) .
\end{aligned}
$$

Hence we have

$$
r(k-t-1) \geqq f(1) \geqq r\{(k-2)-(r-1)(t-1)\} .
$$

Any two vertices in $H_{j}$ are joined together otherwise there would be a claw of order $r+1$. Thus $H_{j}$ is a clique.

If we put $H_{j}^{*}=H_{j}-\left(A_{j} \cup P\right)$, then $H_{j}^{*}$ consists of exactly those vertices of $T$ which are joined to $A_{j}$ but to no other vertex of $S$. Hence $H_{1}^{*}, H_{2}^{*}, \cdots, H_{r}^{*}$ are disjoint sets and the total number of vertices in these sets is $f(1)$, which satisfies (9.1). 
Now there is a unique grand clique $K_{j}$ containing $A_{j}$ and $P(j=$ $1,2, \cdots, r)$. The number of vertices in $K_{j}$ cannot be less than the number of vertices in $H_{j}$. If possible let $\left|K_{j}\right|<\left|H_{j}\right|$. Since $K_{j}$ is a grand clique it follows that $H_{j}$ is a major clique and is contained in some grand clique $K_{j}^{\prime}$. Since $A_{j}$ and $P$ are contained in $K_{j}$ and $K_{j}^{\prime}$, they must coincide. Hence $K_{j}$ contains $H_{j}$ which contradicts. $\left|K_{j}\right|<\left|H_{j}\right|$.

Now consider the $r$ grand cliques $K_{1}, K_{2}, \cdots, K_{r}$. Then $K_{1}-P$, $K_{2}-P, \cdots, K_{r}-P$ are disjoint. For if $K_{i}-P$ and $K_{j}-P(i \neq j)$. have a common point $Q$, then $K_{i}$ and $K_{j}$ would coincide and would contain both $A_{i}$ and $A_{j}$ which is impossible since $A_{i}$ is not joined to. $A_{j}$. Now

$$
\begin{aligned}
& \left|K_{1}-P\right|+\left|K_{2}-P\right|+\cdots+\left|K_{r}-P\right| \\
& \quad \geqq\left|H_{1}-P\right|+\left|H_{2}-P\right|+\cdots+\left|H_{r}-P\right| \\
& \quad=r+\left|H_{1}^{*}\right|+\left|H_{2}^{*}\right|+\cdots+\left|H_{r}^{*}\right| \\
& \quad=r+f(1) \\
& \quad \geqq r\{(k-1)-(r-1)(t-1)\} .
\end{aligned}
$$

If possible suppose there is another grand clique $K_{r}+1$ containing $P$. The vertices in $K_{r+1}-P$ must be disjoint from the vertices in $K_{1}-P, \cdots, K_{r}-P$. Also $K_{r+1}-P$ must have at least $(k-1)-$ $(r-1)^{2}(t-1)$ vertices. If we remember that the number of first. associates of $P$ is $r(k-1)$ we have

$$
\begin{gathered}
r\{(k-1)-(r-1)(t-1)\}+(k-1)-(r-1)^{2}(t-1) \leqq r(k-1) . \\
k \leqq 1+(r-1)(2 r-1)(t-1) .
\end{gathered}
$$

Hence $k \leqq q(r, t)$, which gives a contradiction. This finally proves: our lemma.

COROLlary 1. The hypothesis of the Lemma may be replaced by (i) $k>\rho(r, t)$ and (ii) there exists no claw of order $r+1$ in $G$.

This follows from Lemma (9.5), and the inequality $\rho(r, t) \geqq$ $q(r, t)$.

CoRollary 2. The hypothesis of the lemma may be replaced by $k>p(r, t)$.

This follows from Corollary 1, Lemma (8.3), and the inequality $p(r, t) \geqq \rho(r, t)$.

Theorem (9.1). Let $G$ be a pseudo-geometric graph $(r, k, t) . \quad$ If 
(i) $k>q(r, t)$,

(ii) two distinct vertices of $G$ are contained in utmost one grand clique,

(iii) there exists no claw of order $r+1$ in $G$; then $G$ is geometrisable $(r, k, t)$.

TheOREM (9.2). Let $G$ be a pseudo-geometric graph $(r, k, t)$. If

(i) $k>\rho(r, t)$,

(ii) there exists no claw of order $r+1$ in $G$; then $G$ is geometrisable $(r, k, t)$.

THeOREM (9.3). Let $G$ be a pseudo-geometric graph $(r, k, t)$. If $k>p(r, t)$, then $G$ is geometrisable $(r, k, t)$.

If we take the set of grand cliques of $G$, as the set of $\S 6$, then Lemmas (9.5) and (9.6), together with their corollaries show that the axioms $A^{* 1} 1$ and $A^{*} 2$ are satisfied, under the conditions of any of the Theorems (9.1), (9.2), (9.3). The result now follows from Theorem (6.2).

10. The uniqueness of the triangular association scheme for $n>8$. Consider a pseudo-triangular graph with characteristic $n$, which is a pseudo-geometric graph with characteristics $(2, n-1,2)$ and with parameter (7.4), (7.5). In this case $r=2, t=2$ and the function $p(r, t)$ given by (8.4) is equal to 7 . Hence from Theorem (9.3) the graph is geometrisable $(2, n-1,2)$ if $n-1>7$, i.e. $n>8$.

Now $v=n(n-1) / 2$ and $r=2$. Thus each point occurs in exactly two lines. Given any point $P$ not contained in a line $b$, the two lines $m_{1}$ and $m_{2}$ containing $P$, must both intersect $b$, since $r=t=2$. Hence any two lines intersect in a unique point. If we designate the lines by the numbers $1,2, \cdots, n$; then we may make a $(1,1)$ correspondence between points and the unordered number of pairs $(i, j)$, $i \neq j, i, j=1,2, \cdots, n$, where the point corresponding to $(i, j)$ is the intersection of the lines $i$ and $j$. If we now take an $n \times n$ square and write down in the cells $(i, j)$ and $(j, i)$ the treatment corresponding to the unordered pair $(i, j)$, then clearly the points occurring in the same row (or same column) are those occurring in the same line (see Fig. 1 for the case $n=5$ ). Thus the association relations between the vertices of the graph will be exhibited in the form known as the triangular scheme for $n>8$. This result was first obtained by Connor [9]. Of course when we use design of experiments language the vertices of the graph or points are treatments.

Shrikhande [21] has proved the uniqueness of the triangular scheme for $n=5,6$ and Hoffman [13] and Chang [6] have proved the same for $n=7$. Both Hoffman [14] and Chang [7] have shown 
that for $n=8$, the parameters (7.4), (7.5) do not completely determine the scheme. There are three other possible schemes with the same parameters besides the triangular. This may be expressed as follows: There are four non-isomorphic strongly regular graphs with parameters

$$
n_{1}=12, \quad n_{2}=15, \quad p_{11}^{1}=6, p_{11}^{2}=4
$$

only one of which is geometrisable $(2,7,2)$.

Consider a BIB design

$$
\begin{aligned}
& v^{*}=\frac{1}{2}(n-1)(n-2), \quad b^{*}=\frac{1}{2} n(n-1), \quad r^{*}=n, \\
& k^{*}=n-2, \quad \lambda^{*}=2
\end{aligned}
$$

Hall and Connor [12] have shown that if this design exists then it can be embedded in a symmetric BIB design

$$
v_{0}=b_{0}=\frac{1}{2} n(n-1)+1, \quad r_{0}=k_{0}=n, \quad \lambda_{0}=2 .
$$

Their proof does not cover the case $n=8$, for which Connor [10] separately showed that the design (9.1) does not exist.

Shrikhande [22], has proved the Hall-Connor theorem for the case $n \neq 8$ by using the uniqueness of the triangular scheme for $n \neq 8$. It is interesting to observe that $n=8$, the case not covered in Hall and Connor's entirely different proof, is exactly the case when the parameters (7.4), (7.5) do not uniquely characterize the scheme as triangular.

11. Theorems of Shrikhande, Bruck and Mesner on the uniqueness of the $L_{r}$ scheme. Consider $G_{N}$ as a pseudo-net graph with characteristic $(r, k)$ or the corresponding association scheme with parameters (7.1), (7.2). Since $t=r-1$ in this case, $G_{N}$ is geometrisable $(r, k, r-1)$ if

$$
k>p(r, r-1)=\frac{1}{2}(r-1)\left(r^{3}-r^{2}+r+2\right) .
$$

In particular if $r=2$, this reduces to $k>4$.

In the case $r=2$, the geometry consists of two sets of parallel lines. Each parallel class contains $k$ lines, and each line contains $k$ points. Lines of the same class do not intersect. Lines of different. classes intersect in a point. Thus each point is uniquely determined as the intersection of one line from each class. We can number the lines of each class $1,2, \cdots, k$; and we can number the points or vertices of the graph $1,2, \cdots, k^{2}$. We now take a $k \times k$ square and identify the $i$ th line of the first class with the $i$ th row, the $j$ th line of the second class with the $j$ th column, and the cell $(i, j)$ with the 
point which is intersection of the line $i$ of the first class, with the line $j$ of the second class, then the association relations between the vertices of the graph are exhibited as an $L_{2}$ scheme. This proves the uniqueness of the $L_{2}$ scheme for $n>4$ a result obtained by Shrikhande [19] and by Mesner [15].

In the general case the geometry consists of a set of $k^{2}$ points (the vertices of $G_{N}$ ) and $r$ classes of parallel lines, each class containing $k$ lines. Lines of the same class do not intersect. Lines of different classes intersect in one point. Let the parallel classes be designated by $(R),(C),\left(U_{1}\right), \cdots,\left(U_{r-2}\right)$. To the $k$ lines within each class we assign the symbols $1,2, \cdots, k$. Each point is uniquely given by the intersection of a line of $(R)$, with a line of $(C)$. Hence as in the case $r=2$, the lines of $(R)$ may be identified with the rows, and the lines of $(C)$ with the columns of a $k \times k$ square. Then the intersection of the line of $(R)$ and the line of $(C)$ is identified with the cell $(i, j)$. If in each cell $(i, j)$ we put the number of the line of $\left(U_{a}\right)$ which passes through the point corresponding to the cell, we get Latin squares $L_{\alpha}(\alpha=1,2, \cdots, r-2)$ and the Latin squares $L_{1}, L_{2}, \cdots, L_{r-2}$ are mutually orthogonal. Two points (cells) are first associates if they lie in the same row, same column or correspond to the same letter of the same Latin square. Thus the association relations between the points or vertices of $G_{N}$ can be exhibited by the $L_{r}$ scheme defined by Bose and Shimamoto [4]. Thus the $L_{r}$ scheme with parameters $(7.1)-(7.2)$ is unique (up to type) if (11.1) holds. It is necessary to add the words up to type, since there may be many non-isomorphic sets of $r-2$ mutually orthogonal Latin squares. This result is implicit in Bruck's paper [5]. A slightly weaker result was proved by Mesner [15].

12. The SLB scheme and the general uniqueness theorem. Let us consider the SLB scheme or the pseudo-SLB graph with characteristics $(r, k)$ for which the parameters are given by (7.6), (7.7). Then Theorem (9.3) states that the graph is geometrisable $(r, k, r)$ if

$$
k>\frac{1}{2} r\left(r^{3}-r^{2}+r+1\right) .
$$

In the language of designs this would mean that if there is an association scheme with parameters (7.6), (7.7) then if (12.1) holds the association relations can be exhibited by the dual of a BIB design (with $r_{0}=k$ and $k_{0}=r, \lambda_{0}=1$ ) so that the first associates are exactly those which occur together in a block of this dual and the second associates are those which do not occur together in a block of this dual. Thus (7.6), (7.7) determine the structure of the association scheme up to type. It is necessary to add the words up to type 
since there will exist in general non-isomorphic BIB designs with $\left(r_{0}=k, k_{0}=r, \lambda_{0}=1\right)$ and their duals will automatically be non-isomorphic. When (12.1) does not hold we cannot say that their will exist a dual of a BIB design i.e. a partial geometry $(r, k, r)$, whose structure will exhibit the association relations.

In general then we can say that if we have a pseudo-geometric association scheme with parameters (4.1), (4.2), then if

$$
k>p(r, t)=\frac{1}{2}\left[r(r-1)+t(r+1)\left(r^{2}-2 r+2\right)\right] .
$$

the association structure can be exhibited by means of a partial geometry $(r, k, t)$, the first associates being those treatments which correspond to points on a line of the geometry. Such schemes may called geometric schemes. Thus when (12.2) is true, the association scheme will be determined up to type, for there will exist non-isomorphic partial geometries with the same parameters $r, k, t$. This may be regarded as a generalized uniqueness theorem. When (12.2) is not true very little is known except for schemes which have the same parameters as the triangular scheme or the $L_{2}$ scheme $(r=2$, $k, t=1)$. These two cases have fully investigated.

\section{A general embedding theorem.}

Theorem (13.1). Given a PBIB design $\left(r, k, \lambda_{1}, \lambda_{2}\right), \lambda_{1}>\lambda_{2}$ based on a strongly regular graph $G$ (association scheme) with parameters

$$
\begin{aligned}
& n_{1}=(d-1)(k-1)(k-t) / t, \quad n_{2}=d(k-1), \\
& p_{11}^{1}=[(d-1)(k-1)(k-t)-d(k-t-1)-t] / t, \\
& \quad p_{11}^{2}=(d-1)(k-t)(k-t-1) / t .
\end{aligned}
$$

We can extend the design by adding new blocks, containing the same treatments, in such a way that the extended design is a balanced incomplete block (BIB) design with $r_{0}=r+d\left(\lambda_{1}-\lambda_{2}\right)$ replications, block size $k$ and in which every pair of treatments occur together in $\lambda_{1}$ blocks, provided that

$$
k>p(d, t)=\frac{1}{2}\left[d(d-1)+t(d+1)\left(d^{2}-2 d+2\right)\right] .
$$

Let $G^{*}$ be the complementary of $G$, i.e. $G^{*}$ is the graph with the same vertices as $G$, but with the relation of adjacency reversed, i.e. just those vertices in $G^{*}$ are joined which were unjoined in $G$. This means that first associates become second associates and vice versa. The parameters of $G^{*}$ are obtained from $G$ by interchanging the subscripts and superscripts 1 and 2 . Hence for $G^{*}$

$$
n_{1}^{*}=d(k-1), \quad n_{2}^{*}=(d-1)(k-1)(k-t) / t,
$$




$$
\begin{array}{r}
p_{22}^{2 *}=[(d-1)(k-1)(k-t)-d(k-t-1)-t] / t \\
p_{22}^{1 *}=(d-1)(k-t)(k-t-1) / t
\end{array}
$$

Using the identities (2.3), (2.4), we find that

$$
p_{11}^{1 *}=(t-1)(d-1)+k-2, \quad p_{11}^{2 *}=d t .
$$

Hence $G^{*}$ is pseudo-geometric with characteristics $(d, k, t)$.

In view of (13.3) it follows from theorem (9.3) that $G^{*}$ is geometrisable $(d, k, t)$. From (4.1) the geometry has $d[(d-1)(k-1)+t] / t$ blocks, and every pair of treatments which were second associates in the original PBIB design occur once in the new blocks. If we add these new blocks repeated $\lambda_{1}-\lambda_{2}$ times to the original blocks then each pair occurs $\lambda_{1}$ times and each treatment occurs $r+d\left(\lambda_{1}-\lambda_{2}\right)$ times. This proves our theorem.

We shall now derive from this the embedding theorem on orthogonal Latin squares due to Shrikhande and Bruck.

In Theorem (13.1) take $t=d-1$, then $G^{*}$ is a pseudo-geometric graph $(d, k, d-1)$ i.e. a pseudo-net graph. If

$$
k>p(d, d-1)=\frac{1}{2}(d-1)\left(d^{3}-d^{2}+d+2\right),
$$

it is geometrisable.

Also let us take $r=k+1-d, \lambda_{1}=1, \lambda_{2}=0$. Then the PBIB design becomes the design $(k+1-d, k, 1,0)$ based on the strongly regular graph with parameters (7.1), (7.2). This is easy to check by substituting $d=k+1-r$ in (13.1), (13.2) and noting that they reduce to (7.1), (7.2). Hence the PBIB design is a net of degree $k+1-d$, or a lattice design with $r=k+1-d$ and block size $k$. Hence the extended design is a BIB design with $r+d$ i.e. $k+1$ replications in which every pair of treatments occurs in one block. This is an affine plane of order $k$. Hence we have

Theorem (13.2A). A lattice design (or a net) with $r=k+1-d$ and block size $k$ can be completed to an affine plane

$$
v_{0}=k^{2}, \quad b_{0}=k(k+1), \quad r_{0}=k+1, \quad k_{0}=k, \quad \lambda_{0}=1,
$$

by adding kd new blocks, if $k>\frac{1}{2}(d-1)\left(d^{3}-d^{2}+d+2\right)$.

Now we have already noticed the equivalence of a lattice design with $r$ replications and block size $k$, with a set of $r-2$ mutually orthogonal Latin squares of order $k$. Since an affine plane of order $k$ can be regarded as a lattice with $k+1$ replications, Theorem (13.2A) may alternatively be stated as

THEOREM (13.2B). If there exist $k-1-d$ mutually orthogonal 
Latin squares of order $k$, it is possible to get a complete set of $k-1$ mutually orthogonal Latin squares, by adding $d$ new suitably chosen squares, provided that $k>\frac{1}{2}(d-1)\left(d^{3}-d^{2}+d+2\right)$.

The case $d=2$, was first obtained by Shrikhande [20] and the: general case was obtained by Bruck [5].

\section{REFERENCES}

1. R. C. Bose and W. H. Clatworthy, Some classes of partially balanced designs, Ann. Math. Stat., 26 (1955), 212-232.

2. R. C. Bose and D. M. Mesner, On linear associative algebras corresponding to association schemes of partially balanced designs, Ann. Math. Stat., 30 (1959), 21-38.

3. R. C. Bose and K. R. Nair, Partially balanced incomplete block designs, Sankhya, 4. (1939), 337-372.

4. R. C. Bose and T. Shimamoto, Classification and analysis of partially balanced incomplete block designs, with two associate classes, J. Amer. Stat. Assn., 47 (1952), 151184.

5. R. H. Bruck, Finite nets II. Uniqueness and imbedding, Pacific J. Math., 13. (1963), 421-457.

6. Chang Li-chien, The uniqueness and non-uniqueness of the triangular association. schemes, Science Record, Math., New Ser., 3 (1959), 604-613.

7. - Association schemes of partially balanced designs with parameters $v=28$, $n_{1}=12, n_{2}=15$ and $p_{11}^{2}=4$. Science Record, Math., New Ser. 4 (1960), 12-18.

8. W. H. Clatworthy, A geometrical configuration wich is a partially balanced incomplete block design, Proc. Amer. Math. Soc., 5 (1954), 47-55.

9. W. S. Connor, The uniqueness of the triangular association scheme, Ann. Math. Stat., 29 (1958), 262-266.

10. - On the structure of balanced incomplete block designs, _Ann. Math. Stat., 23 (1952), 57-71.

11. W. S. Connor and W. H. Clatworthy, Some theorems for partially balanced designs, 25 (1954), 100-112.

12. Marsnall Hall and W. S. Connor, An embedding theorem for balanced incomplete block designs, Can. J. Math., 6 (1953), 35-41.

13. A. J. Hoffman, On the uniqueness of the triangular association scheme, Ann. Math. Stat., 31 (1960), 492-497.

14. On the exceptional case in a characterization of the arcs of a complete graph, IBM J., 4 (1960), 487-496.

15. D. M. Mesner, An investigation of certain combinatorial properties of partially balanced incomplete block experimental designs and association schemes, with a detailed study of designs of Latin square and related types, unpublished doctoral thesis, Michigan State University, 1956.

16. E. J. F. Primrose, Quadratics in finite geometries, Proc. Camb. Phil. Soc. 47 (1951), 299-304.

17. D. K. Ray-Chaudhuri, Some results on quadrics in finite projective geometry, Can. J. Math., 14 (1962), 129-138.

18. - Application of the geometry of quadrics for constructing PBIB designs, Ann. Math. Stat., 33 (1962), 1175-1186.

19. S. S. Shrikhande, The uniqueness of the $L_{2}$ 'association scheme Ann. Math. Stat., 30 (1959), 781-798.

20. — A note on mutually orthogonal Latin squares, Sankhya, 23 (1961), $115-116$. 
21. S. S. Shrikhande, On a characterization of the triangular association scheme, Ann. Math. Stat., 30 (1959), 39-47.

22. — Relations between certain incomplete block designs, Contributions to probability and statistics, Essays in honor of Harold Hotelling, Stanford U. Press (1960), 388-395.

23. — On the dual of some balanced incomplete block designs, Biometrics, 8 (1952), 66-72.

24. F. Yates, Lattice squares, J. Ag. So., 30 (1940), 672-687.

UNIVERSity OF NoRth CAROLINA AND UNIVERSity OF Geneva 



\title{
PACIFIC JOURNAL OF MATHEMATICS
}

\author{
EDITORS
}

\author{
Ralph S. Phillips \\ Stanford University \\ Stanford, California \\ M. G. Arsove \\ University of Washington \\ Seattle 5 , Washington
}

\author{
J. DugunduI \\ University of Southern California \\ Los Angeles 7, California
}

Lowell J. Paige

University of California

Los Angeles 24, California

\section{ASSOCIATE EDITORS}

E. F. BECKENBACH

T. M. CHERRY
D. DERRY
M. OHTSUKA

H. L. ROYDEN

E. SPANIER
E. G. STRAUS

F. WOLF

\section{SUPPORTING INSTITUTIONS}

UNIVERSITY OF BRITISH COLUMBIA CALIFORNIA INSTITUTE OF TECHNOLOGY UNIVERSITY OF CALIFORNIA MONTANA STATE UNIVERSITY UNIVERSITY OF NEVADA NEW MEXICO STATE UNIVERSITY OREGON STATE UNIVERSITY UNIVERSITY OF OREGON OSAKA UNIVERSITY UNIVERSITY OF SOUTHERN CALIFORNIA
STANFORD UNIVERSITY

UNIVERSITY OF TOKYO

UNIVERSITY OF UTAH

WASHINGTON STATE UNIVERSITY

UNIVERSITY OF WASHINGTON

AMERICAN MATHEMATICAL SOCIETY CALIFORNIA RESEARCH CORPORATION SPACE TECHNOLOGY LABORATORIES NAVAL ORDNANCE TEST STATION

Mathematical papers intended for publication in the Pacific Journal of Mathematics should be typewritten (double spaced), and the author should keep a complete copy. Manuscripts may be sent to any one of the four editors. All other communications to the editors should be addressed to the managing editor, L. J. Paige at the University of California, Los Angeles 24, California.

50 reprints per author of each article are furnished free of charge; additional copies may be obtained at cost in multiples of 50 .

The Pacific Journal of Mathematics is published quarterly, in March, June, September, and December. Effective with Volume 13 the price per volume (4 numbers) is $\$ 18.00$; single issues, $\$ 5.00$. Special price for current issues to individual faculty members of supporting institutions and to individual members of the American Mathematical Society: $\$ 8.00$ per volume; single issues \$2.50. Back numbers are available.

Subscriptions, orders for back numbers, and changes of address should be sent to Pacific Journal of Mathematics, 103 Highland Boulevard, Berkeley 8, California.

Printed at Kokusai Bunken Insatsusha (International Academic Printing Co., Ltd.), No. 6, 2-chome, Fujimi-cho, Chiyoda-ku, Tokyo, Japan.

PUBLISHED BY PACIFIC JOURNAL OF MATHEMATICS, A NON-PROFIT CORPORATION

The Supporting Institutions listed above contribute to the cost of publication of this Journal, but they are not owners or publishers and have no responsibility for its content or policies. 


\section{Pacific Journal of Mathematics}

\section{Vol. 13, No. 2 \\ April, 1963}

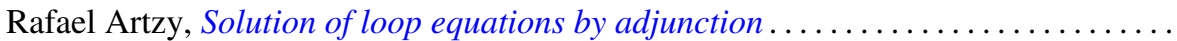

Earl Robert Berkson, A characterization of scalar type operators on reflexive

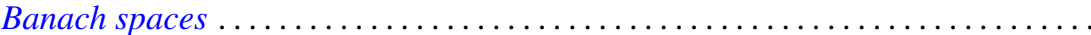

Mario Borelli, Divisorial varieties

365

Raj Chandra Bose, Strongly regular graphs, partial geometries and partially

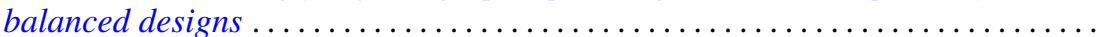

389

R. H. Bruck, Finite nets. II. Uniqueness and imbedding ............... 421

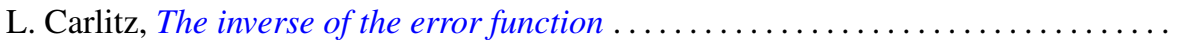

Robert Wayne Carroll, Some degenerate Cauchy problems with operator

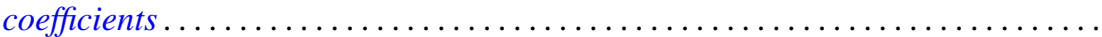

Michael P. Drazin and Emilie Virginia Haynsworth, A theorem on matrices of 0 's

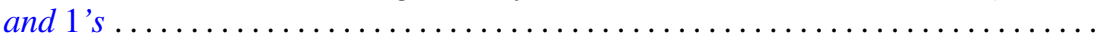

Lawrence Carl Eggan and Eugene A. Maier, On complex approximation .......... James Michael Gardner Fell, Weak containment and Kronecker products of group

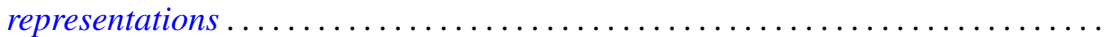

Paul Chase Fife, Schauder estimates under incomplete Hölder continuity

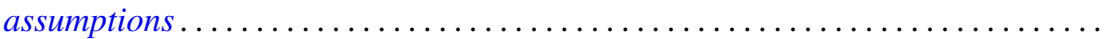

Shaul Foguel, Powers of a contraction in Hilbert space ...................

Neal Eugene Foland, The structure of the orbits and their limit sets in continuous

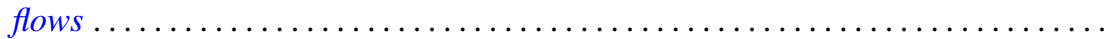

Frank John Forelli, Jr., Analytic measures . . . . . . . . . . . . . . . . . . . . . 563

Robert William Gilmer, Jr., On a classical theorem of Noether in ideal theory ....... P. R. Halmos and Jack E. McLaughlin, Partial isometries .

Albert Emerson Hurd, Maximum modulus algebras and local approximation in

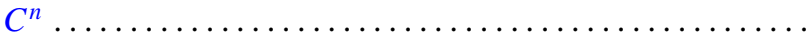

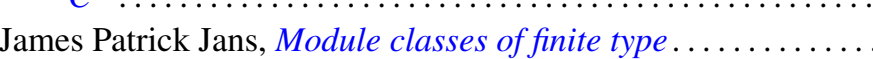

Betty Kvarda, On densities of sets of lattice points ...

H. Larcher, A geometric characterization for a class of discontinuous groups of linear fractional transformations .

John W. Moon and Leo Moser, Simple paths on polyhedra .

T. S. Motzkin and Ernst Gabor Straus, Representation of a point of a set as sum of

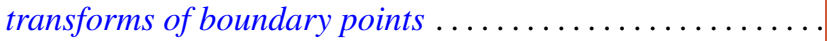

Rajakularaman Ponnuswami Pakshirajan, An analogue of Kolmogorov's three-series theorem for abstract random variables ...............

Robert Ralph Phelps, Čebyšev subspaces of finite codimension in $C(X)$...

James Dolan Reid, On subgroups of an Abelian group maximal disjoint from a given subgroup ...

William T. Reid, Riccati matrix differential equations and non-oscillation criteria for associated linear differential systems .................

Georg Johann Rieger, Some theorems on prime ideals in algebraic number fields ...

Gene Fuerst Rose and Joseph Silbert Ullian, Approximations of functions on the

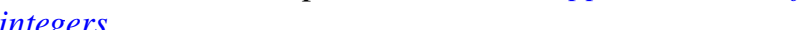

F. J. Sansone, Combinatorial functions and regressive isols . . . . . . . . . . 703

Leo Sario, On locally meromorphic functions with single-valued moduli . . . . . . . 709

Takayuki Tamura, Semigroups and their subsemigroup lattices.

Pui-kei Wong, Existence and asymptotic behavior of proper solutions of a class of second-order nonlinear differential equations . . ........... 